\title{
INTERPOLATION SEARCH: A MEMOIZED APPROACH
}

\author{
Deepti Verma $^{1}$ and Dr. Kshama Paithankar ${ }^{2}$
}

\begin{abstract}
While dealing with a large amount of data, methods/ techniques are to be used to perform searching appropriate data. Time is the core concern while evaluating performance of these techniques. While addressing this issue, for further time minimization, an efficient method as an extension of binary search namely Interpolation Search technique has been emerged. Observations led to move towards optimization of the performance of this algorithm. Literature reveals optimization of time in various algorithms achieved through an approach of memoization. Hence, interpolation search technique is implemented with memorization in this paper. The results indicate a significant time reduction and thus achieve the objective of improving performance of interpolation search technique.
\end{abstract}

Keywords: Interpolation Search, Memoization, Execution Time.

\section{INTRODUCTION}

Searching is the process of finding a particular item in an available collection of items. Literature and study material is evident of existence of many searching methods/techniques especially in the area of computer science. While dealing with a large amount of data, such process is the core to access the selective items in processing. Mainly, linear search, binary search etc. are available to perform searching of data. The performance of any searching algorithm is evaluated primarily in terms of time required to seek data item or specific value. It has been observed that this seek time is a major issue with existing searching algorithms. However, binary searching algorithm proves better among the available algorithms. This issue has been taken up for further time minimization and hence an efficient method as an extended version of binary search namely Interpolation Search technique has been emerged.

Verification of the performance of Interpolation Search led to move towards optimization of the performance of this algorithm. It has been observed that for optimization of time in various algorithms, memoization has proved to be a method to achieve this objective[1]. Memoization is also known as function caching. It was first introduced in 1968 in the context of Artificial Intelligence [2]. It is a way for machines to learn from past experiences. Here, it is proposed to develop an algorithm of interpolation search MemIPS() with memoized approach to optimize the time of element searching.

\footnotetext{
${ }^{1}$ Shri Vaishnav ${ }^{S M}$ Institute of Management, Indore

${ }^{2}$ Shri Vaishnav ${ }^{S M}$ Institute of Management, Indore
} 
The concept of memoization is described in section 2. Section 3 includes the formal description of Non-memoized Interpolation search function NIPS(). Formal description of memoized Interpolation Search function $\operatorname{MemIPS()}$ is presented in section 4. Section 5 deals with case study to show performance of MemIPS() and NIPS(). Section 6 covers discussion on results in different cases. Finally, we end-up with the conclusion and future scope in section 7 .

\section{MEMOIZATION}

Memoization proves to be a technique for reducing the execution time of program [3]. Memoized function stores the output and provides it when user calls same function again with same value [4]. In other words, using memoization programs could "recall" previous computations and thus avoid repeated work [5][6]. The key idea behind this is to speed up the execution of a function by maintaining the cache of its previous computations and look-up into the cache instead of computing data repeatedly.

Normally, if functions are being called more than once, its computational code is executed and the results of each iteration are to be stored. It consumes time and affects the memory utilization as well. Excluding for the first time, in every next step, results are looked up in cache resulting in reducing redundancy, computation time and thus improvement of performance and efficiency [7][8].

\section{FORMAL DESCRIPTION OF NIPS()}

Interpolation search is a classical method for searching through ordered random data[9]. It is retrieving a desired record by key in an ordered file by using the value of the key and the statistical distribution of the keys. It works on Divide and Conquer method[10]. Here, the algorithm NIPS() is presented.

\section{Algorithm NIPS()}

$/ * \operatorname{arr}[]$ represent the data set*/

$/ * \mathrm{n} 1$ contains the size of data set*/

$/ *$ item is an integer type variable which holds the key value which we want to search */

/*mid is a class level integer variable*/

/*last and first are the integer type variables to hold the first and last position value*/

Start

Step 1: int inpoSearch (int arr[],int n1,int item)

/*Function declaration with the parameters of data set and key value and size of data set.*/

Step 2: first $=0$;

$$
\text { last }=\mathrm{n} 1-1
$$

$/ *$ initializes first with 0 and size of data set is stored in last.*/

Step 3: while(first <= last)

$$
\begin{aligned}
& \{\text { mid }=\text { first }+(\text { last }- \text { first }) *((\text { item } \quad-\operatorname{arr}[\text { first }]) /(\operatorname{arr}[\text { last }]-\operatorname{arr}[\text { first }])) \\
& \text { if }(\operatorname{arr}[\text { mid }]==\text { item }) \\
& \{\text { return } 1 ;\}
\end{aligned}
$$


/*To find the mid, and if this element matches the item then return $1 * /$

Step 4: $\quad$ if $($ item $<\operatorname{arr}[$ mid] $)$

last $=\operatorname{mid}-1$;

else

first $=\operatorname{mid}+1$;

/*Updating values of first and last as per the condition. */

Step 5: if (first > last)

return 0 ;

/*returning the value 0 if no search*/

End

\section{FORMAL DESCRIPTION OF MemIPS()}

In the previous section, regular NIPS() has been discussed. Now in this section, $\operatorname{MemIPS()}$ algorithm is being presented. MemIPS() accepts the values entered by the user and verifies the output. The value found is stored in variable and whenever needed, this variable will be extracted as store value. The process continues till the result is available.

Algorithm MemIPS()

$/ * \mathrm{v}$ is an integer type variable*/

$/ *$ st 1 and ed 1 are integer type local variables*/

$/ * b$ is an integer type local variable used to retrieve output*/

Start

Step 1: st $1=$ Systemtime

$/ *$ to stores starting time $* /$

Step 3: b=d1.memoIps();

/* Retrieving output */

ed1=Systemtime;

$/ *$ getting ending time */

Step 4: $\quad$ tot $1=($ ed1-st 1$)$;

Message ("Memo Time "+tot1);

/*Showing total time with memoization*/

Step 5: if $(v==1)$

Message ("Value Found $="+b$ );

/*When the value is found then show the output with time*/

End

\section{CASE STUDY}

The performance of proposed algorithm MemIPS() and that of NIPS() has been evaluated using three cases. Case 1 includes the study for data size 10 whereas Case 2 deals with data size as 25. Data size 50 was considered for study in Case 3. These different cases have been studied for the NIPS() as well.

\section{Case 1: Study for data size 10}


Here, the performance of NIPS() and MemIPS() is discussed with the list containing 10 values. Table-1 illustrates the performance in terms of time whereas Figure- 1 represents the trend of time including that memoized function improve the performance consistently.

Table-1: Dataset of 10 values (Values from 10 to 100)

\begin{tabular}{|l|l|l|l|}
\hline Search & $\begin{array}{l}\text { Non } \\
\text { Memoize } \\
\text { V Time }\end{array}$ & $\begin{array}{l}\text { Memoize } \\
\text { d Time }\end{array}$ & $\begin{array}{l}\text { Differen } \\
\text { ce }\end{array}$ \\
\hline 10 & 2265 & 1359 & 906 \\
\hline 20 & 2718 & 1359 & 1359 \\
\hline 30 & 3170 & 1358 & 1812 \\
\hline 40 & 3171 & 1359 & 1812 \\
\hline 50 & 3623 & 1359 & 2264 \\
\hline 60 & 3623 & 1359 & 2264 \\
\hline 70 & 4982 & 1359 & 3623 \\
\hline 80 & 4529 & 1359 & 3170 \\
\hline 90 & 4528 & 1359 & 3169 \\
\hline 100 & 2264 & 1358 & 906 \\
\hline $\begin{array}{l}\text { Not } \\
\text { Found }\end{array}$ & 2717 & 1812 & 905 \\
\hline
\end{tabular}

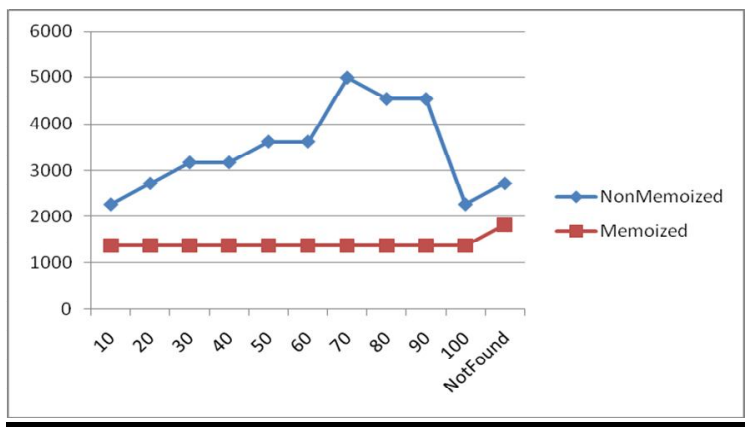

Figure-1: Performance of MemIPS() vs NIPS() in Case 1.

\section{Case 2: Study for data size 25}

In this Case, the list of dataset containing 25 values is experimented with proposed MemIPS() and NIPS() as well as shown in Table-2. Similarly, Figure-2 represents the trend of time. In this Case increasing the size of the dataset also results in improving time and thus the performance of algorithm using memoization.

Table-2: Dataset of 25 values (Values from 10 to 250)

\begin{tabular}{|l|l|l|l|l|}
\hline $\begin{array}{l}\text { Search } \\
\text { Value }\end{array}$ & $\begin{array}{l}\text { Non } \\
\text { Memoized } \\
\text { Time }\end{array}$ & $\begin{array}{l}\text { Mem } \\
\text { oized } \\
\text { Time }\end{array}$ & $\begin{array}{l}\text { Differen } \\
\text { ce }\end{array}$ & Range \\
\hline
\end{tabular}




\begin{tabular}{|c|c|c|c|c|}
\hline 50 & 7699 & 1358 & 6341 & $10-100$ \\
\hline 80 & 4076 & 1359 & 2717 & $10-100$ \\
\hline 120 & 4982 & 1359 & 3623 & $\begin{array}{l}100- \\
150\end{array}$ \\
\hline 140 & 5888 & 1359 & 4529 & $\begin{array}{l}100- \\
150\end{array}$ \\
\hline 170 & 5888 & 1811 & 4077 & $\begin{array}{l}150- \\
200\end{array}$ \\
\hline 190 & 6340 & 1359 & 4981 & $\begin{array}{l}150- \\
200\end{array}$ \\
\hline 220 & 7693 & 1358 & 6335 & $\begin{array}{l}200- \\
250\end{array}$ \\
\hline 240 & 7699 & 1359 & 6340 & $\begin{array}{l}200- \\
250\end{array}$ \\
\hline 110 & 4076 & 1358 & 2718 & random \\
\hline 210 & 6793 & 1358 & 5435 & random \\
\hline Not Found & 7699 & 1812 & 5887 & \\
\hline
\end{tabular}

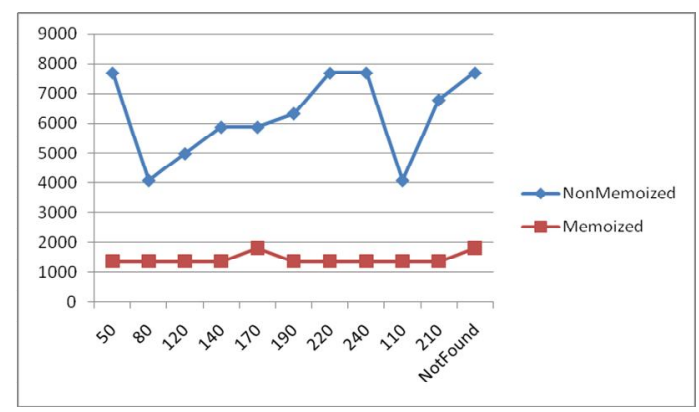

Figure-2: Performance of MemIPS() vs NIPS() in Case 2.

\section{Case 3: Study for data size 50}

A list containing 50 values for implementing NIPS() and MemIPS() is used in this Case. Table-3 highlights the outcome in terms of time and Figure-3 represents the trend of this time. Here, it is observed that the performance of MemIPS() still follows the trend of optimization of time to improve performance of searching.

Table-3: Dataset of 50 values (Values 10 to 500)

\begin{tabular}{|l|l|l|l|l|}
\hline $\begin{array}{l}\text { Search } \\
\text { Value }\end{array}$ & $\begin{array}{l}\text { Non } \\
\text { Memoized } \\
\text { Time }\end{array}$ & $\begin{array}{l}\text { Mem oized } \\
\text { Time }\end{array}$ & $\begin{array}{l}\text { Diffe } \\
\text { rence }\end{array}$ & range \\
\hline 50 & 3170 & 1359 & 1811 & $10-100$ \\
\hline 80 & 4982 & 1359 & 3623 & $10-100$ \\
\hline 120 & 4982 & 1359 & 3623 & $100-200$ \\
\hline 180 & 5887 & 1359 & 4528 & $100-200$ \\
\hline
\end{tabular}




\begin{tabular}{|l|l|l|l|l|}
\hline 210 & 6794 & 1359 & 5435 & $200-300$ \\
\hline 250 & 7699 & 1359 & 6340 & $200-300$ \\
\hline 350 & 9511 & 1358 & 8153 & $300-400$ \\
\hline 370 & 9963 & 1359 & 8604 & $300-400$ \\
\hline 420 & 11322 & 1358 & 9964 & $400-500$ \\
\hline 460 & 11775 & 1359 & 10416 & $400-500$ \\
\hline 300 & 8152 & 1359 & 6793 & random \\
\hline 440 & 11775 & 1359 & 10416 & random \\
\hline $\begin{array}{l}\text { NotFo } \\
\text { und }\end{array}$ & 2717 & 1359 & 1358 & NotFound \\
\hline
\end{tabular}

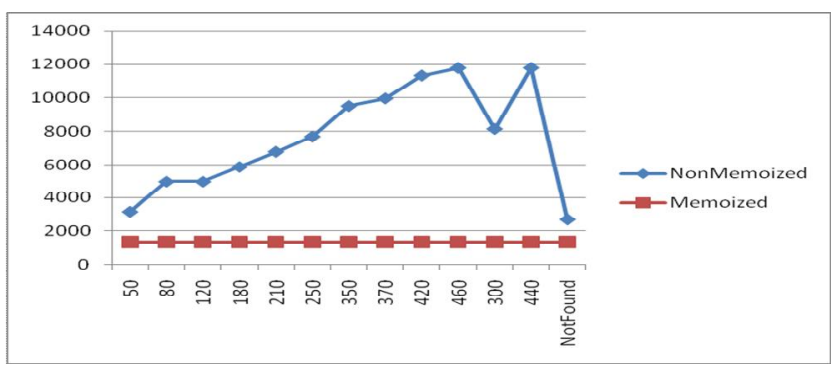

Figure-3: Performance of MemIPS() vs NIPS() in Case 3.

\section{DISCUSSION}

With the help of three cases, it has been noticed that MemIPS() really improves the performance of searching as compare to regular NIPS(). For instance, in Case 1 the time recorded to search the first element is $2265 \mathrm{~ms}$ using $\operatorname{NIPS()}$ whereas using $\operatorname{MemIPS}()$ it is noted $1359 \mathrm{~ms}$ thereby reducing the time by $906 \mathrm{~ms}$ clearly as shown in Table- 4 .

Table-4: Comparative Performance in different Cases

\begin{tabular}{|l|l|l|l|l|l|}
\hline $\begin{array}{l}\text { cas } \\
\text { eas }\end{array}$ & Index & $\begin{array}{l}\text { Search } \\
\text { Value }\end{array}$ & $\begin{array}{l}\text { Non } \\
\text { Memoized } \\
\text { Time }\end{array}$ & $\begin{array}{l}\text { Memoiz } \\
\text { ed Time }\end{array}$ & $\begin{array}{l}\text { Differe } \\
\text { nce }\end{array}$ \\
\hline \multirow{2}{*}{1} & First & 10 & 2265 & 1359 & 906 \\
\cline { 2 - 6 } & Mid & 50 & 3623 & 1359 & 2264 \\
\cline { 2 - 6 } & Last & 100 & 2264 & 1358 & 906 \\
\hline \multirow{2}{*}{2} & First & 50 & 7699 & 1358 & 6341 \\
\cline { 2 - 6 } & Mid & 170 & 5888 & 1811 & 4077 \\
\cline { 2 - 6 } & Last & 210 & 6793 & 1358 & 5435 \\
\hline \multirow{3}{*}{3} & First & 50 & 3170 & 1359 & 1811 \\
\cline { 2 - 6 } & Mid & 210 & 6794 & 1359 & 5435 \\
\cline { 2 - 6 } & Last & 460 & 11775 & 1359 & 10416 \\
\hline
\end{tabular}




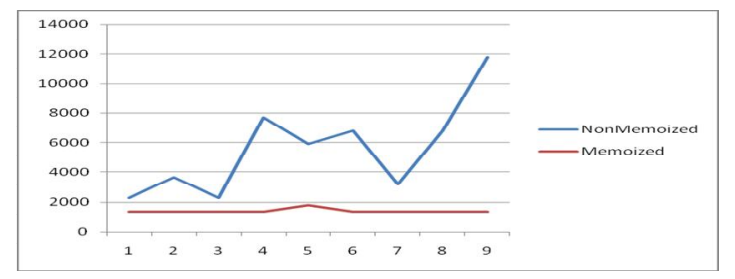

Figure-4: Cumulative bracket of $\operatorname{MemIPS}()$ and NIPS().

Similarly, for mid element, time recorded is $3623 \mathrm{~ms}$ using NIPS() and in MemIPS() it is $1359 \mathrm{~ms}$. Here, time is reducing by $2264 \mathrm{~ms}$ and in the case of last element the searching time is recorded using NIPS() is $2264 \mathrm{~ms}$ where as using MemIPS() it is computed as $1358 \mathrm{~ms}$ thereby reducing by $906 \mathrm{~ms}$. This trend is observed in Case 2 and Case 3 also as represented by Figure-4. Presently, the performance of MemIPS() is evaluated for first, middle and last elements of the lists. However, the trends of performance indicate that it may surely be applicable for the element at any position in the list.

\section{CONCLUSION}

In this paper, the proposed MemIPS() proves to be a effective algorithm that optimizes the execution time with optimum utilization of memory and thus improving the overall performance of the Regular Interpolation Search algorithm. However, there exists scope of further enhancement in many aspects. Presently a single data type has been used where as multiple data types may be worked in order to improve the performance proportionally. Further, data types long, float and double may be used leading to more accuracy of result.

\section{REFERENCES}

[1] Purey, J., Paithankar, K., Memoization: a Technique to optimize Performance of Searching, National Conference on "Challenges of Globalization and Strategies for Competitiveness" Shri Vaishnav Institute of Management, Indore, January, 2015, pp 486-491.

[2] Norvig,P. "Techniques for Automatic Memoization with Applications to Context-Free Parsing", University of California, Volume 17,Issue1, March 1991, pp 91-98 .

[3] Pfeffer,A. "Sampling with Memoization", School of Engineering and Applied Sciences, Harvard University, 2007.

[4] Crockford, D. “Java Script The Good Parts”, O'Reilly, May 2008, pp 44-45.

[5] Ziarek, L; Sivaramakrishnan,K.C.and Jagannathan, S. "Partial Memoization of Concurrency and Communication", Department of Computer Science Purdue University, Proceedings of the $14^{\text {th }}$ ACM SIGPLAN International Conference on Functional Programming. Edinburgh, Scotland, ACM, pp. 161172.

[6] Acar,U.A.; Blelloch,G.E. and Harper,R. "Selective Memoization", School of Computer Science Carnegie Mellon University Pittsburgh, PA 15213, 2003.

[7] Brown,.D. and Cook, W.R. "Monadic Memoization Mixins", Department of Computer Sciences, University of Texas at Austin, 2006.

[8] Purey,J and Muley,K. "Auto Response Memoization using JAVA", National Conference on Emerging Technologies in Electronics, Mechanical and Computer Engineering (ETEMC) April 2010.

[9] Graham, S.L. and Rivest, R.L. "Interpolation Search A Log LogN Search", 3 July 1993.

[10] Demaine, E.D.; Jones, T. and Patrascu,M. "Interpolation Search for Non-Independent Data", Proceedings of the $15^{\text {th }}$ Annual ACM-SIAM Symposium on Discrete Algorithms (SODA) , 2004, pp 529-530. 